\title{
Evaluation of Postprandial Glucose Excursion Using a Novel Minimally Invasive Glucose Area-Under-the-Curve Monitoring System
}

\author{
Sachi Kuranuki, $\mathrm{PhD}^{1^{*} \text {, Toshiyuki Sato, MSc}}{ }^{2}$, Seiki Okada, $\mathrm{MSc}^{2}$, \\ Samiko Hosoya, $\mathrm{BSc}^{2}$, Akinobu Seko, BSc ${ }^{3}$, Kaya Sugihara, $\mathrm{MSc}^{3}$ and \\ Teiji Nakamura, $\mathbf{P h D}^{\mathbf{1}}$ \\ ${ }^{1}$ Faculty of Health \& Social Work, Kanagawa University of Human Services, \\ Kanagawa, Japan \\ ${ }^{2}$ Central Research Laboratories, \\ ${ }^{3}$ New Business Development Group, Sysmex Corporation, Kobe, Japan
}

Submitted March 2013. Accepted for publication August 2013.

\begin{abstract}
Objective: To develop a minimally invasive interstitial fluid extraction technology (MIET) to monitor postprandial glucose area under the curve (AUC) without blood sampling, we evaluated the accuracy of glucose AUC measured by MIET and compared with that by blood sampling after food intake. Methods: Interstitial fluid glucose AUC (IG-AUC) following consumption of 6 different types of foods was measured by MIET. MIET consisted of stamping microneedle arrays, placing hydrogel patches on the areas, and calculating IG-AUC based on glucose levels in the hydrogels. Glycemic index (GI) was determined using IG-AUC and reference AUC measured by blood sampling. Results: IG-AUC strongly correlated with reference AUC $(R=0.91)$, and GI determined using IG-AUC showed good correlation with that determined by reference AUC ( $R=0.88$ ). Conclusions: IG-AUC obtained by MIET can accurately predict the postprandial glucose excursion without blood sampling. In addition, feasibility of GI measurement by MIET was confirmed.
\end{abstract}

Keywords: glucose monitor, glycemic index, postprandial hyperglycemia, interstitial fluid, nutrition education

\section{INTRODUCTION}

It is well known that insufficient glucose control in patients with diabetes results in cardiovascular diseases, blindness, kidney failure, and other complications. The DECODE study showed that there is an increased risk of cardiovascular diseases in patients whose postprandial glucose, rather than fasting plasma glucose (FPG), is high, suggesting that it is important to manage postprandial glucose excursion [1]. Since

*Corresponding author: Sachi Kuranuki, Faculty of Health \& Social Work, Kanagawa University of Human Services, 1-10-1 Heiseicho, Yokosuka 238-8522, Japan. Phone: +81-46-828-2664. Fax: +81-46-828-2665. Email: kuranuki-s@kuhs.ac.jp. Other authors: sato.toshiyuki@sysmex.co.jp; okada.seiki@sysmex.co.jp; hosoya.samiko@sysmex.co.jp; seko.akinobu@sysmex.co.jp; sugihara.kaya@sysmex.co.jp; nakamura-t @kuhs.ac.jp. 
postprandial glucose excursion is related to carbohydrate intake, "carbohydrate counting" and "glycemic index (GI) use," which are educational methods for food selection, are widely used for glucose control in patients with diabetes. Carbohydrate counting considers the amount of carbohydrate required for glucose excursion, and has been mainly used in patients with type 1 diabetes to calculate the amount of carbohydrate in food relative to insulin treatment. Carbohydrate counting has recently become a tool for food selection in type 2 diabetes, and some reports have demonstrated its usefulness in glucose control $[2,3]$.

On the other hand, GI is an index that considers quality of carbohydrate [4]. GI is a comparison between the increase in glucose level after consuming a food containing $50 \mathrm{~g}$ of carbohydrate with the increase after consuming bread containing $50 \mathrm{~g}$ of carbohydrate and is affected by protein, fat, and fiber content, as well as the type of carbohydrate in the food [5]. Postprandial glucose excursions are largely affected by the GI of the food $[6,7]$. While GI is mainly measured as a value for a single meal, most diets consist of multiple meals. Although the GI of multiple meals can be calculated from that of each individual meal, it has often been noted that this does not indicate actual glucose excursion after the consumption of these meals [8]. In addition to contents of the meal, glucose excursion depends on the patient's general condition. Therefore, it is necessary to measure actual postprandial glucose excursion to gain a better understanding of glycemic status of an individual.

Self-monitoring of blood glucose (SMBG) is widely adopted in patients with diabetes receiving insulin treatment to control their blood glucose levels [9]. SMBG has been reported to result in good postprandial glucose control in patients with type 2 diabetes, which in turn results in reduced hemoglobin A1C (HbA1c) levels in these patients [10]. Although measuring blood glucose after each meal is useful in gaining a better understanding of the glycemic status, it usually requires repeated blood sampling which inevitably involves pain. Recently developed continuous glucose-monitoring technology provides useful information but can be burdensome for type 2 diabetes patients because of the blood sampling required for calibration and the subcutaneous needle insertion.

Therefore, we developed a novel system that uses minimally invasive interstitial fluid extraction technology (MIET) to estimate postprandial glucose excursion without blood sampling. The procedures for measurement of glucose area under the curve (AUC) of blood glucose vs. time curve are shown in Figure 1: (1) A plastic microneedle array is stamped on the forearm skin to enhance the interstitial fluid (ISF) extraction rate. (2) A hydrogel patch is placed on the pretreated area to collect ISF. The rate of glucose extraction from ISF is proportional to the ISF glucose (IG) concentration level. Therefore, the reservoir contains glucose proportional to interstitial fluid glucose (IG)-AUC for the examination period. Simultaneously, the level of accumulated sodium ions is measured for use as an internal standard, rather than calibration by blood sampling. (3) Extracted glucose and sodium ion levels in the hydrogel are measured to calculate IG-AUC for the examination period. In this system, ISF extraction rate is important because glucose extraction rate depends on both the blood glucose level and the ISF extraction rate. The ISF extraction rate, 


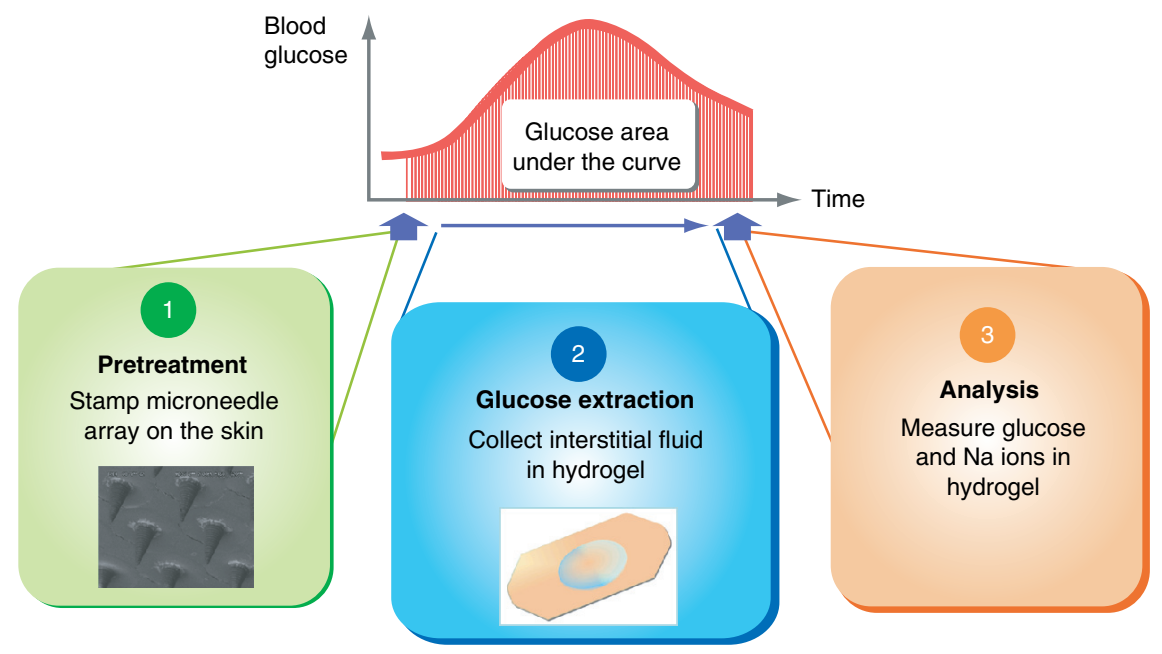

Figure 1. Procedures of the minimally invasive glucose AUC monitoring system.

which depends on the micropore size, can be estimated by the sodium ion extraction rate because sodium ion levels do not fluctuate much in individuals. Calibration using the sodium ion extraction rate enables us to calculate IG-AUC without blood sampling. Blood sampling is unnecessary with our system because postprandial glucose excursions are easily estimated by the glucose AUC, which corresponds to the total increase in postprandial glucose levels. Conversely, accurate estimation of postprandial glucose excursions using SMBG is difficult because it requires a large number of blood samples.

A proof-of-principle study to examine the underlying concept of our technology was conducted and reported by Sato et al. [11]. The accuracy of the measurement system was then evaluated by measuring IG-AUC during an oral glucose tolerance test (OGTT) administered to individuals with and without diabetes in order to compare IGAUC with reference plasma glucose PG-AUC values [12]. In addition to high correlation between the two, the advantage of painless nature of IG-AUG technique over PG-AUG was confirmed.

The objective of this study is to evaluate the accuracy of glucose AUC measured by MIET and compared with that by blood sampling after food consumption. In order to determine its potential application with regard to nutrition education, we evaluated the performance of MIET during relatively small glucose excursions in healthy volunteers who ate 5 types of confectionery items containing $50 \mathrm{~g}$ of carbohydrate and different percentages of protein and fat. In addition, GI for each food consumption was calculated using IG-AUC measured by MIET to evaluate the possibility of convenient GI determination. 


\section{METHODS}

\subsection{Measurement Protocol}

Glucose excursions were measured using SMBG and MIET in 5 healthy male subjects (20-40 years, BMI $21 \pm 3 \mathrm{~kg} / \mathrm{m}^{2}$ ) who consumed the test and standard foods (Table 1). To measure IG-AUC by MIET, the forearm skin was wiped with an antiseptic, and a microneedle applicator was employed to stamp microneedle arrays and collect ISF at 4 sites simultaneously. Next, hydrogel patches were placed on the 4 pretreated areas to collect ISF. Two hydrogel patches were also placed on untreated areas for 2 and $3 \mathrm{~h}$, respectively, to detect sweat level. Two patches on the pretreated areas and 1 patch on the untreated areas were removed $2 \mathrm{~h}$ after food intake to analyze the ISF constituents and to calculate IG-AUC. The remaining hydrogel patches were removed $3 \mathrm{~h}$ after food intake and analyzed in the same manner. Capillary blood glucose levels (taken from a finger) were measured before and 15, 30, 45, 60, 90, 120, and 180 min after test food intake by an SMBG device (Freestyle Freedom, Nipro, Japan) to calculate blood glucose AUC (BG-AUC) as reference AUC and GI. All foods were served with sugar-free tea and consumed within $15 \mathrm{~min}$.

On the basis of ease of preparation and content stability, 5 typical confectionary items, each containing $50 \mathrm{~g}$ of total carbohydrate (with $>95 \%$ available carbohydrates), were selected as the test foods. Rice containing $50 \mathrm{~g}$ of carbohydrate was selected as the standard food for GI calculation. Detailed information on the confectionary items examined and the standard food is shown in Table 1. All tests including the standard

Table 1. Energy levels and constituents of each test food and standard food

\begin{tabular}{|c|c|c|c|c|c|c|c|c|c|c|c|}
\hline \multirow[b]{2}{*}{ Item } & \multicolumn{2}{|r|}{ Menu } & \multirow{2}{*}{$\frac{\text { Weight }}{(\mathrm{g})}$} & \multicolumn{2}{|c|}{ Energy } & \multicolumn{3}{|c|}{ PFC weight } & \multicolumn{3}{|c|}{ PFC weight ratic } \\
\hline & Content & Name & & (kcal) & $(\mathbf{k J})$ & $\begin{array}{l}\mathbf{P} \\
(\mathrm{g})\end{array}$ & $\begin{array}{c}\mathbf{F} \\
(\mathrm{g})\end{array}$ & $\begin{array}{l}\mathrm{C}^{\mathrm{C}} \\
(\mathrm{g})\end{array}$ & $\begin{array}{c}P \\
(\%)\end{array}$ & $\begin{array}{c}F \\
(\%)\end{array}$ & $\begin{array}{c}\mathrm{C} \\
(\%)\end{array}$ \\
\hline $\mathrm{a}$ & $\begin{array}{l}\text { Rice } \\
\text { (standard) }\end{array}$ & $\begin{array}{l}\text { Satou no gohan } \\
\text { (Sato food) }\end{array}$ & 150 & 216 & 904 & 3.2 & 0.5 & 49.7 & 5.9 & 2.1 & 92 \\
\hline $\mathrm{b}$ & Chocolate & Kitcut (Nestle) & 90 & 495 & 2,071 & 6.5 & 29 & 52.5 & 5.2 & 53 & 42 \\
\hline c & $\begin{array}{l}\text { Rice } \\
\text { cracker }\end{array}$ & $\begin{array}{l}\text { Onigiri Senbei } \\
\text { (Masuya) }\end{array}$ & 70 & 335 & 1,402 & 4.5 & 13.5 & 48 & 5.4 & 37 & 58 \\
\hline $\mathrm{d}$ & $\begin{array}{l}\text { Ice } \\
\text { cream }\end{array}$ & $\begin{array}{l}\text { Cookie and Cream } \\
\text { (Haagen-Dazs) }\end{array}$ & 240 & 538 & 2,251 & 9.8 & 34 & 47.8 & 7.3 & 57 & 36 \\
\hline e & $\begin{array}{l}\text { Bean } \\
\text { jelly }\end{array}$ & $\begin{array}{l}\text { Ogura Yokan } \\
\text { Yoruno-ume } \\
\text { (Toraya) }\end{array}$ & 75 & 218 & 912 & 2.7 & 0.9 & 51 & 4.8 & 3.6 & 92 \\
\hline$f$ & Pudding & $\begin{array}{l}\text { Baked Pudding } \\
\text { (Ohayo milk) }\end{array}$ & 280 & 374 & 1,565 & 13 & 15 & 46.6 & 14 & 36 & 50 \\
\hline
\end{tabular}

$\mathrm{P}=$ Protein; $\mathrm{F}=$ Fat $\mathrm{C}=$ Carbohydrate Bean jelly is a jelly made of beans and sugars, which is a popular confectionary in Japan and is called Yokan. 
food test were performed once only for each subject. The ethics committee of Sysmex Corporation, Kobe, Japan, approved the study protocol. Written informed consent was received from all subjects.

\subsection{Apparatus and Materials}

The microneedle array was made from polycarbonate and covered approximately $50 \mathrm{~mm}^{2}$, with 305 needles of $0.3 \mathrm{~mm}$ length each (Figure 1, panel 1). The applicator for microneedle stamping of the skin was a spring-action, handheld system with a stamping speed of approximately $6 \mathrm{~m} / \mathrm{s}$. The hydrogel patch, comprising hydrogel and adhesive tape (KP; Nichiban, Tokyo, Japan) was used for ISF collection (Figure 1, panel 2); the hydrogel contained polyvinyl alcohol with $2 \% \mathrm{KCl}$ solvent. The reagent for glucose analysis consisted of 4 enzymes and 1 dye in a phosphate buffer solution. Each $0.1 \mathrm{~mL}$ of the phosphate buffer solution contained 2.6 U glucose oxidase (Wako Pure Chemical Industries, Osaka, Japan), $0.023 \mathrm{U}$ mutarotase (Wako Pure Chemical Industries), 0.25 $\mathrm{U}$ peroxidase (Wako Pure Chemical Industries), $0.49 \mathrm{U}$ ascorbic acid oxidase (Wako Pure Chemical Industries), and $0.016 \mathrm{~mL}$ Amplex red solution (Molecular Probes, Eugene, OR, USA).

\subsection{Procedures for Glucose/Sodium Ion Analyses}

The hydrogel was separated from the adhesive tape before analysis of the extract, and was then immersed overnight in $5 \mathrm{~mL}$ pure water to extract glucose and sodium ions. For glucose measurement, $0.1 \mathrm{~mL}$ of the sample solution was mixed with 0.1 $\mathrm{mL}$ of the reagent. After $60 \mathrm{~min}$ of incubation, the fluorescent intensity of Amplex red was measured using a fluorescence plate reader (GENios, TECAN, Japan). Sodium ion levels were analyzed using the DX-500 ion chromatography system (Dionex, IL, USA).

\subsection{Data Analysis}

IG-AUC was calculated from the measured mass of glucose $M_{g l u}(\mathrm{nmol})$ and sodium ions $M_{n a}(\mathrm{nmol})$ in the hydrogel. The details and principles of data analysis have been described previously $[11,12]$. In this study, $\alpha$ and $\beta$ were $8.26 \times 10^{-5}$ and $3.35 \times 10^{-3}$

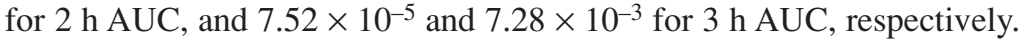

The increase in AUC (iAUC) and GI of each food for each subject was calculated from IG-AUC as follows:

$$
\begin{gathered}
\mathrm{i} A U C(m g \cdot h / d l)=I G-A U C(2 h)-B G(\text { fasting }) \times 2 h \\
G I(\text { test food })=\mathrm{iAUC}(\text { test food }) / i A U C(\text { standard food }) \times 100
\end{gathered}
$$

GI of each food was calculated as the average GI for the subjects. Reference GI was calculated by SMBG using standard methods [13].

Student paired t-tests were employed to analyze the data. Pearson's product-moment correlation coefficients were used to analyze correlations between 2 variables. 


\section{RESULTS}

\subsection{Glucose Profiles}

Figure 2 shows the postprandial glucose profiles for all foods consumed. Rice (a), rice cracker (c), and bean jelly (e) produced relatively greater glucose excursions, whereas chocolate (b), ice cream (d), and pudding (f) produced relatively smaller glucose excursions. As reported previously [6,7], glucose excursions varied widely for different types of food even when the carbohydrate level was constant. This may be due to several factors such as protein and fat content, as well as the forms of carbohydrate in the food. Glucose levels returned to baseline within $180 \mathrm{~min}$ of all food consumptions.

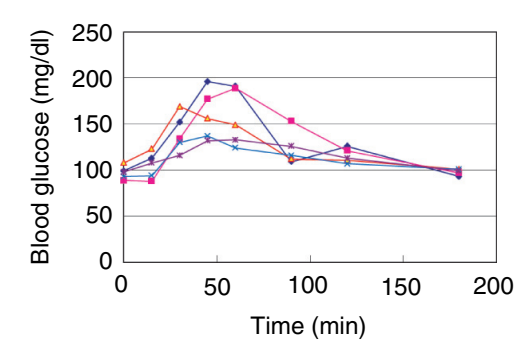

(a) Rice

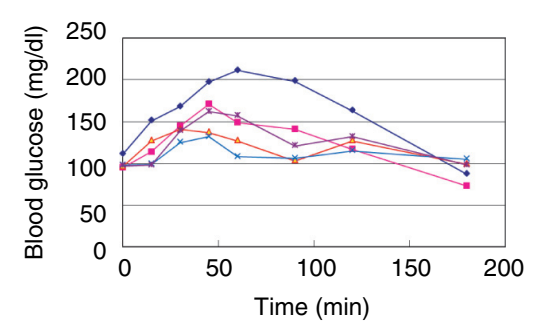

(c) Rice cracker

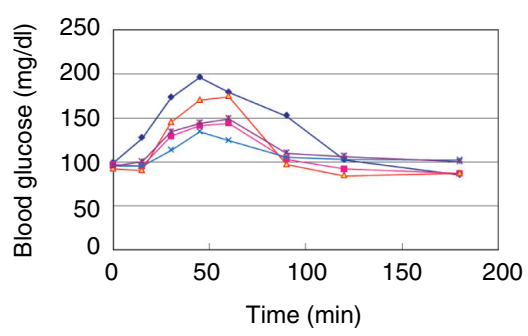

(e) Bean jelly

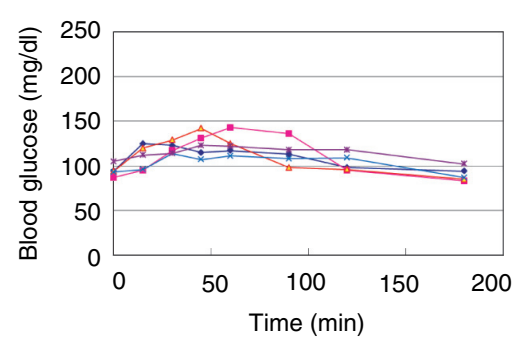

(b) Chocolate

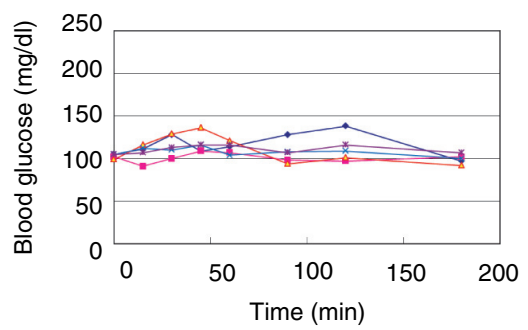

(d) Ice cream

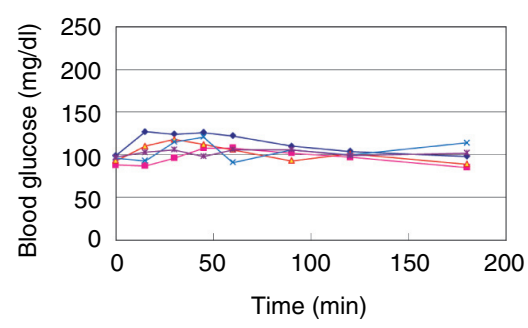

(f) Pudding

Figure 2. Postprandial glucose excursions after intake of (a) rice, (b) chocolate, (c) rice cracker, (d) ice cream, (e) bean jelly, and (f) pudding in the 5 subjects (data indicated by different symbols in different colors). 


\subsection{Correlation Between IG-AUC and Reference BG-AUC}

Figure $3 \mathrm{a}$ shows the correlation between IG-AUC and reference BG-AUC. The correlation coefficient was high $(R=0.91)$ over a wide range $(189-505 \mathrm{mg} \cdot \mathrm{h} / \mathrm{dL})$. The reproducibility of 2 simultaneous IG-AUC measurements was $3.3 \%$ (coefficient of variation, CV), and the mean percentage error from the regression line was $9.1 \%$. These results showed a high accuracy of MIET with relatively small glucose excursions after food consumption.

(a)

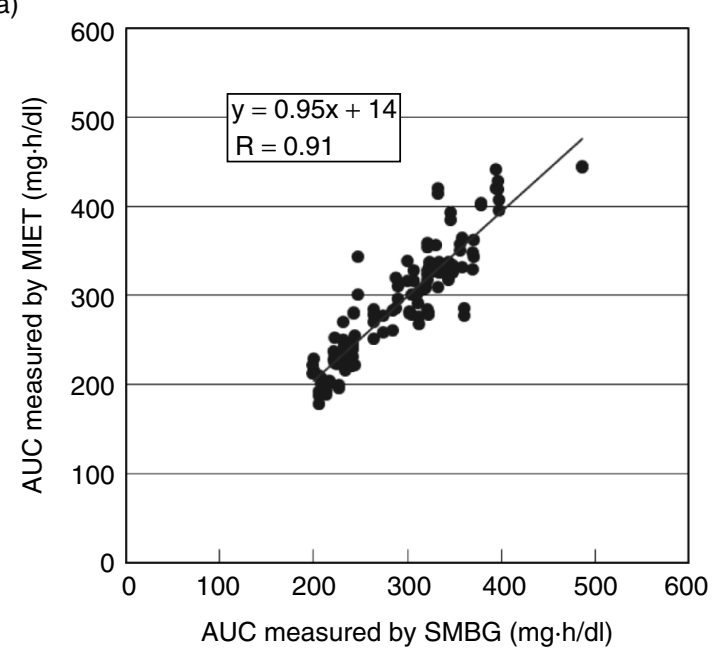

(b)

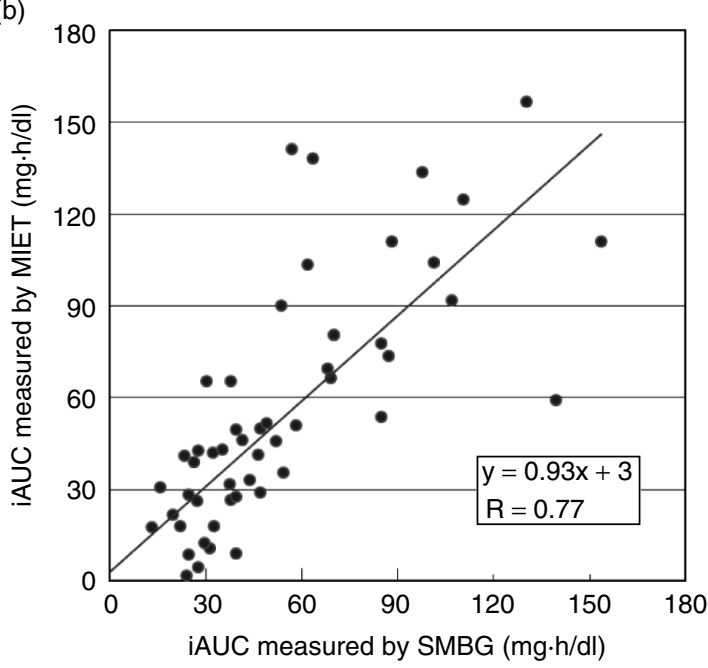

Figure 3. (a) Correlation between AUC data measured by SMBG and MIET. (b) Correlation between iAUC data measured by SMBG and MIET. 
Table 2. Mean AUC and iAUC data for all foods

\begin{tabular}{|c|c|c|c|c|c|c|c|}
\hline \multicolumn{2}{|r|}{ Menu } & \multicolumn{3}{|c|}{ AUC } & \multicolumn{3}{|c|}{ iAUC } \\
\hline Item & Content & MIET \pm SD & $\mathrm{SMBG} \pm \mathrm{SD}$ & $p$ value & $\mathrm{MIET} \pm \mathrm{SD}$ & SMBG \pm SD & $p$ value \\
\hline \multirow[t]{2}{*}{$\mathrm{a}$} & Rice & & & & & & \\
\hline & (standard) & $263 \pm 28$ & $263 \pm 25$ & 1.00 & $68 \pm 34$ & $68 \pm 29$ & 1.00 \\
\hline b & Chocolate & $217 \pm 23$ & $229 \pm 10$ & 0.13 & $27 \pm 29$ & $40 \pm 17$ & 0.13 \\
\hline $\mathrm{c}$ & Rice cracker & $260 \pm 20$ & $274 \pm 53$ & 0.47 & $61 \pm 18$ & $75 \pm 42$ & 0.47 \\
\hline \multirow[t]{2}{*}{ d } & Ice & & & & & & \\
\hline & cream & $219 \pm 10$ & $222 \pm 14$ & 0.75 & $14 \pm 10$ & $16 \pm 13$ & 0.82 \\
\hline \multirow[t]{2}{*}{$\mathrm{e}$} & Bean & & & & & & \\
\hline & jelly & $277 \pm 34$ & $250 \pm 32$ & 0.15 & $85 \pm 36$ & $59 \pm 30$ & 0.15 \\
\hline $\mathrm{f}$ & Pudding & $209 \pm 21$ & $211 \pm 13$ & 0.80 & $20 \pm 21$ & $22 \pm 9$ & 0.85 \\
\hline
\end{tabular}

Figure $3 \mathrm{~b}$ shows the correlation between iAUC data measured by IG-AUC and that measured by SMBG. The correlation coefficient was lower $(\mathrm{R}=0.77)$ than that between AUCs because the ratio of the error levels to the reference levels was magnified by the subtraction of the baseline blood glucose levels from AUC reference levels.

Table 2 shows a summary of AUC and iAUC as measured by MIET and SMBG. No statistically significant differences between AUC and iAUC levels measured by SMBG and MIET were observed.

\subsection{Correlation Between GI as Determined by IG-AUC and Reference GI}

Figure 4 shows the correlation between GI determined by IG-AUC and reference GI $(R=0.88)$, suggesting that our system can accurately predict GI with minimal blood sampling. Although there is significant error in iAUC measured by MIET (Figure 3b), averaging procedures of GI determination for reduction in differences between individuals probably reduce the error levels induced by MIET (Figure 4).

\section{DISCUSSION}

\subsection{Glucose AUC as an Index of Postprandial Glucose Excursion}

In this study, we measured glucose AUC as an index of postprandial glucose excursion. Glucose AUC is considered to represent the complete glucose excursion after food consumption, and is therefore primarily used in patients being treated with either dietrelated therapy or medication for postprandial hyperglycemia, including $\alpha$-glucosidase inhibitors and glinides. In these cases, postprandial glucose excursion by AUC can be used to monitor glycemic control in conjunction with conventional indices such as HbA1c and FPG [14, 15]. This trend is also apparent in the evaluation of recently developed medication, such as incretin-related medicines (e.g., DPP-4 inhibitor or GLP-1 analog) and sodium-glucose cotransporter type $2[16,17]$.

However, although glucose AUC is widely used as an index, there is no clear target for patients with diabetes. Correlations among glucose AUC, glucose levels after a 2-h 


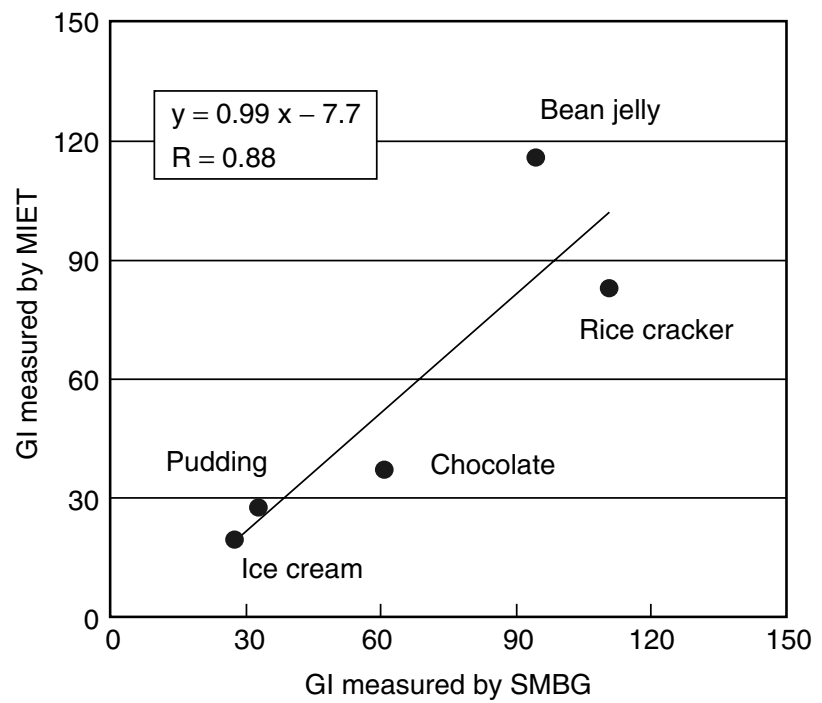

Figure 4. Correlation between $\mathrm{Gl}$ as determined by SMBG and MIET.

load, peak glucose levels, and risk of complications have not been clarified in detail. Further investigation of these parameters will be required to utilize glucose AUC in the daily management of postprandial glucose excursion in patients with diabetes.

\subsection{Usefulness of Glucose AUC Measured by MIET}

It is well known that glucose metabolism is controlled by homeostasis. Therefore, disruption of the metabolic balance is reversed naturally. Hyperglycemia that results from the disruption of glucose metabolism in type 2 diabetes can be modified by glucose management during the early stages. Moreover, glucose management can prevent complications and comorbidities. Hence, it is important for patients to be educated with regard to food selection during the early stages of diabetes [18]. Although GI and carbohydrate counting are used as educational tools with regard to food selection, these methods require calculations for them to be applicable to food selection and changes in dietary habits, making them inconvenient, undesirable, or unfeasible for long-term adoption. Furthermore, monitoring of glucose excursion is required to confirm the effectiveness of these methods. Indeed, postprandial glucose excursion is affected by not only dietary carbohydrate levels but also protein, fat, and fiber levels and the type of carbohydrate in the food, leading to differences in glucose levels predicted by these methods and glucose excursion measured after consumption of multiple foods [19].

Self-monitoring of postprandial blood glucose is valuable as an educational tool in selecting foods that do not induce significant glucose excursion as well as a management tool for glucose control. In fact, it has been reported that HbA1c levels in patients with diabetes decreased after the initiation of SMBG [10]. It was assumed 
that this benefit was due to lifestyle improvements related to the educational effects of SMBG [20]. However, accurate estimation of postprandial hyperglycemia by SMBG is difficult because glucose levels vary greatly with time, and frequent measurements by SMBG cause pain and inconvenience, making it less feasible in daily management.

Glucose AUC measured by MIET has certain benefits compared to that measured by SMBG. For example, the procedure is painless and requires a single measurement to predict total glucose excursion. This enables frequent use of the system in the domestic setting for early-stage diabetes. The first step in current conventional dietary therapy is to determine foods appropriate for the individual patient. Subsequently, dietary therapy is applied to patients' daily life. Utilization of MIET to measure glucose can change this process. Patients may now be readily able to establish an accurate correlation between the effects of their regular diet and postprandial glucose excursion, and can then tailor their dietary therapy based on empirical data.

However, the system still has limitations for home use because the measurement of the contents in the hydrogels needs to be performed in the laboratory, not at home. Development of an easy-to-use system for real time postprandial glucose AUC measurement is necessary to further enhance the effects of the dietary therapy.

\subsection{MIET as a Tool in GI Determination}

The standard GI determination protocol requires 7 repeated measurements of blood glucose level [13]. This number of repeated measurements prevents the ready utilization of GI data for a wide variety of foods, particularly for mixed foods. Our easyto-use system may contribute to deriving GI data for mixed foods served by food companies, groceries, and restaurants, thus helping patients with diabetes to select the appropriate diet for optimal glycemic control.

\subsection{Limitations of Study}

In this study, subjects were all healthy volunteers who had not been diagnosed with diabetes. Further studies are required to confirm the efficacy of AUC measured by MIET as a tool in nutrition education for postprandial glucose management in patients with diabetes.

We measured glucose AUC once only after a food consumption, even though 3 measurements for standard foods would have been more appropriate [13]. We used SMBG with finger sticks for reference blood glucose measurement, but this is not recommended in GI determination because of its low accuracy. In addition, the number of subjects was insufficient, since 10 subjects are necessary for formal GI determination to reduce the individual variability of glucose excursion [13]. In this study, our purpose is to evaluate the correlation between GI measured by SMBG and by MIET, not to measure precise GI for food indexing. A larger number of food varieties should be tested in a future study, in addition to a larger number of subjects. However, to compare AUCs by MIET and reference method, sufficient number of subject would be important to elucidate the equivalence of two methods with enough detection power. 


\section{CONCLUSION}

Results of the present study indicate good correlation between glucose AUC measured by SMBG and that measured by MIET. An easy-to-use glucose monitoring system may be a useful tool in subjects with diabetes to enhance their understanding of the correlation between individual foods and postprandial glucose excursion. Furthermore, the data revealed that it may be feasible to determine GI using glucose AUC measurement by MIET, which may contribute to the promotion of use of GI in patients with diabetes.

\section{CONFLICT OF INTEREST}

S.K. and T.N. received research funding from Sysmex. T.S., S.O., S.H., A.S., and K.S. are employees of Sysmex. No other potential conflicts of interest related to this article are reported.

\section{REFERENCES}

[1] The DECODE study group. European Diabetes Epidemiology Group. Diabetes Epidemiology: Collaborative analysis Of Diagnostic criteria in Europe. Glucose tolerance and mortality: comparison of WHO and American Diabetes Association diagnostic criteria. Lancet, 1999, 354: 617-621.

[2] Mehta, SN, Quinn N, Volkening LK, and Laffel LM. Impact of carbohydrate counting on glycemic control in children with type 1 diabetes. Diabetes Care, 2009, 32:1014-1016.

[3] Gillespie SJ, Kulkarni KD, and Daly AE. Using carbohydrate counting in diabetes clinical practice. $J$ Am Diet Assoc, 1998, 98:897-905.

[4] Burger KN, Beulens JW, van der Schouw YT, Sluijs I, Spijkerman AM, Sluik D, Boeing H, Kaaks R, Teucher B, Dethlefsen C, Overvad K, Tjønneland A, Kyrø C, Barricarte A, Bendinelli B, Krogh V, Tumino R, Sacerdote C, Mattiello A, Nilsson PM, Orho-Melander M, Rolandsson O, Huerta JM, Crowe F, Allen N, Nöthlings U. Dietary fiber, carbohydrate quality and quantity, and mortality risk of individuals with diabetes mellitus. PLoS One, 2012, 7:e43127.

[5] Wolever TM, Miller JB. Sugars and blood glucose control. Am J Clin Nutr, 1995, 62(1 Suppl): 212S221S; discussion 221S-227S.

[6] Jenkins, DJ, Wolever TM, Taylor RH, Barker H, Fielden H, Baldwin JM, Bowling AC, Newman HC, Jenkins AL, and Goff DV. Glycemic index of foods: a physiological basis for carbohydrate exchange. Am J Clin Nutr, 1981, 34:362-366.

[7] Wolever TM, Jenkins DJ, Jenkins AL, and Josse RG. The glycemic index: methodology and clinical implications. Am J Clin Nutr, 1991, 54:846-54.

[8] Gilbertson HR, Thorburn AW, Brand-Miller JC, Chondros P, and Werther GA. Effect of low-glycemicindex dietary advice on dietary quality and food choice in children with type 1 diabetes. Am J Clin Nutr, 2003, 77:83-90.

[9] The Diabetes Control and Complications Trial Research Group. The effect of intensive treatment of diabetes on the development and progression of long-term complications in insulin-dependent diabetes mellitus. N Engl J Med, 1993, 329:977-986.

[10] Clar C, Barnard K, Cummins E, Royle P, and Waugh N. Aberdeen Health Technology Assessment Group. Self-monitoring of blood glucose in type 2 diabetes: systematic review. Health Technol Assess, 2010, 14:1-140.

[11] Sato T, Okada S, Hagino K, Asakura Y, Kikkawa Y, Kojima J, Maekawa Y, Nakajima H, and Asano $\mathrm{K}$. Measurement of glucose area under the curve using minimally invasive interstitial fluid extraction technology: evaluation of glucose monitoring concepts without blood sampling. Diabetes Technol Ther, 2011, 13:1194-1200. 
[12] Sakaguchi K, Hirota Y, Hashimoto N, Ogawa W, Sato T, Okada S, Hagino K, Asakura Y, Kikkawa Y, Kojima J, Maekawa Y, and Nakajima H. A minimally invasive system for glucose area under the curve measurement using interstitial fluid extraction technology; evaluation of the accuracy and usefulness with oral glucose tolerance tests in subject with and without diabetes. Diabetes Technol Ther, 2012, 14:485-491.

[13] Standards Australia. Australian standard glycemic index of food 2007.

[14] Aoki K, Nakamura A, Ito S, Nezu U, Iwasaki T, Takahashi M, Kimura M, and Terauchi Y. Administration of miglitol until 30 min after the start of a meal is effective in type 2 diabetic patients. Diabetes Res Clin Pract., 2007, 78:30-33.

[15] Li J, Tian H, Li Q, Wang N, Wu T, Liu Y, Ni Z, Yu H, Liang J, Luo R, Li Y, and Huang L. Improvement of insulin sensitivity and beta-cell function by nateglinide and repaglinide in type 2 diabetic patients a randomized controlled double-blind and double-dummy multicentre clinical trial. Diabetes Obes Metab, 2007, 9:558-565.

[16] Barrington P, Chien JY, Showalter HD, Schneck K, Cui S, Tibaldi F, Ellis B, and Hardy TA. A 5-week study of the pharmacokinetics and pharmacodynamics of LY2189265, a novel, long-acting glucagonlike peptide-1 analogue, in patients with type 2 diabetes. Diabetes Obes Metab, 2011, 13:426-433.

[17] Polidori D, Sha S, Mudaliar S, Ciaraldi TP, Ghosh A, Vaccaro N, Farrell K, Rothenberg P, and Henry RR. Canagliflozin Lowers Postprandial Glucose and Insulin by Delaying Intestinal Glucose Absorption in Addition to Increasing Urinary Glucose Excretion: Results of a randomized, placebocontrolled study. Diabetes Care 2013.

[18] Lecomte P, Vol S, Cacès E, Lasfargues G, Combe H, Laurent S, and Tichet J. Impaired fasting glycaemia and undiagnosed diabetes: prevalence, cardiovascular and behavioural risk factors. Diabetes Metab, 2002, 28:311-320.

[19] De Natale C, Annuzzi G, Bozzetto L, Mazzarella R, Costabile G, Ciano O, Riccardi G, and Rivellese A.A. Effects of a plant-based high-carbohydrate/high-fiber diet versus high-monounsaturated fat/lowcarbohydrate diet on postprandial lipids in type 2 diabetic patients. Diabetes Care, 2009, 32:2168-2173.

[20] Guerci B, Drouin P, Grangé V, Bougnères P, Fontaine P, Kerlan V, Passa P, Thivolet Ch, Vialettes B, and Charbonnel B; ASIA Group. Self-monitoring of blood glucose significantly improves metabolic control in patients with type 2 diabetes mellitus: the Auto-Surveillance Intervention Active (ASIA) study. Diabetes Metab, 2003, 29:587-594. 


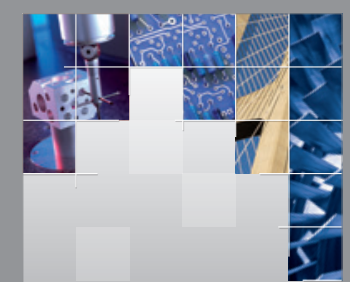

\section{Enfincering}
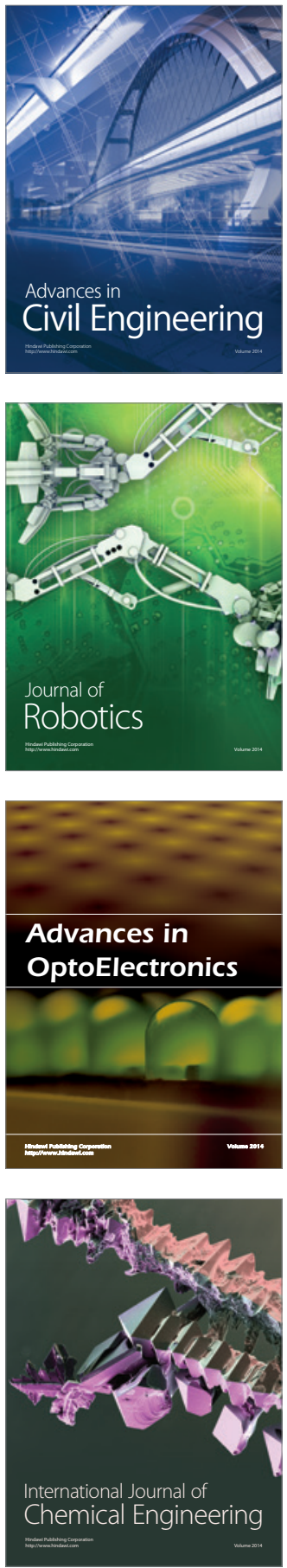

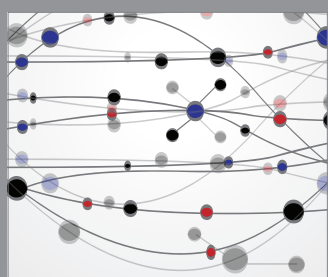

The Scientific World Journal

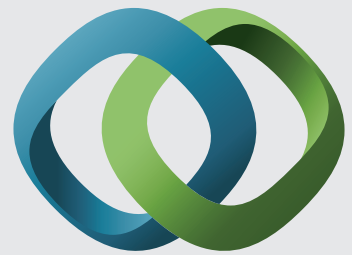

\section{Hindawi}

Submit your manuscripts at

http://www.hindawi.com
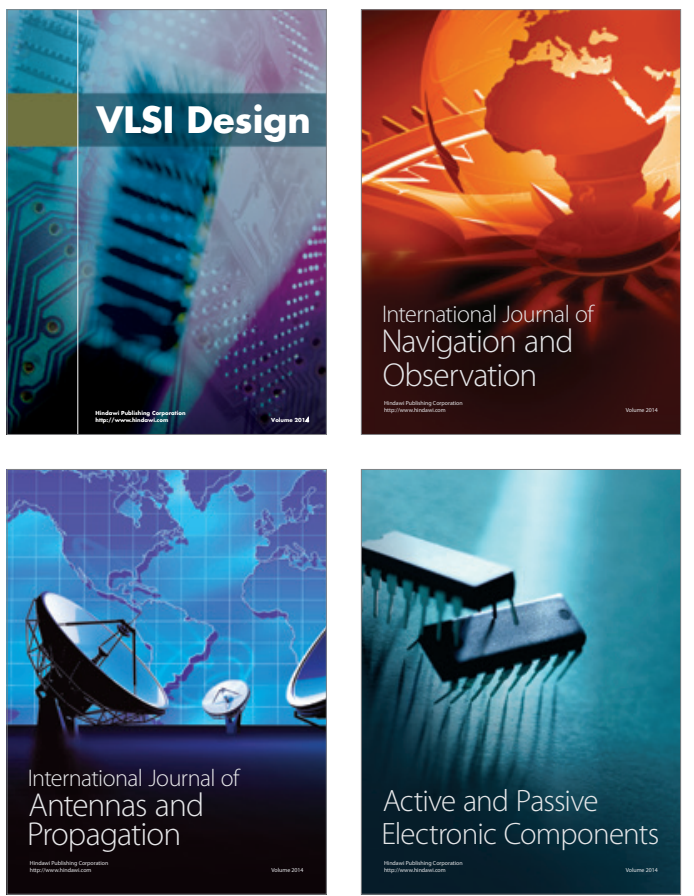
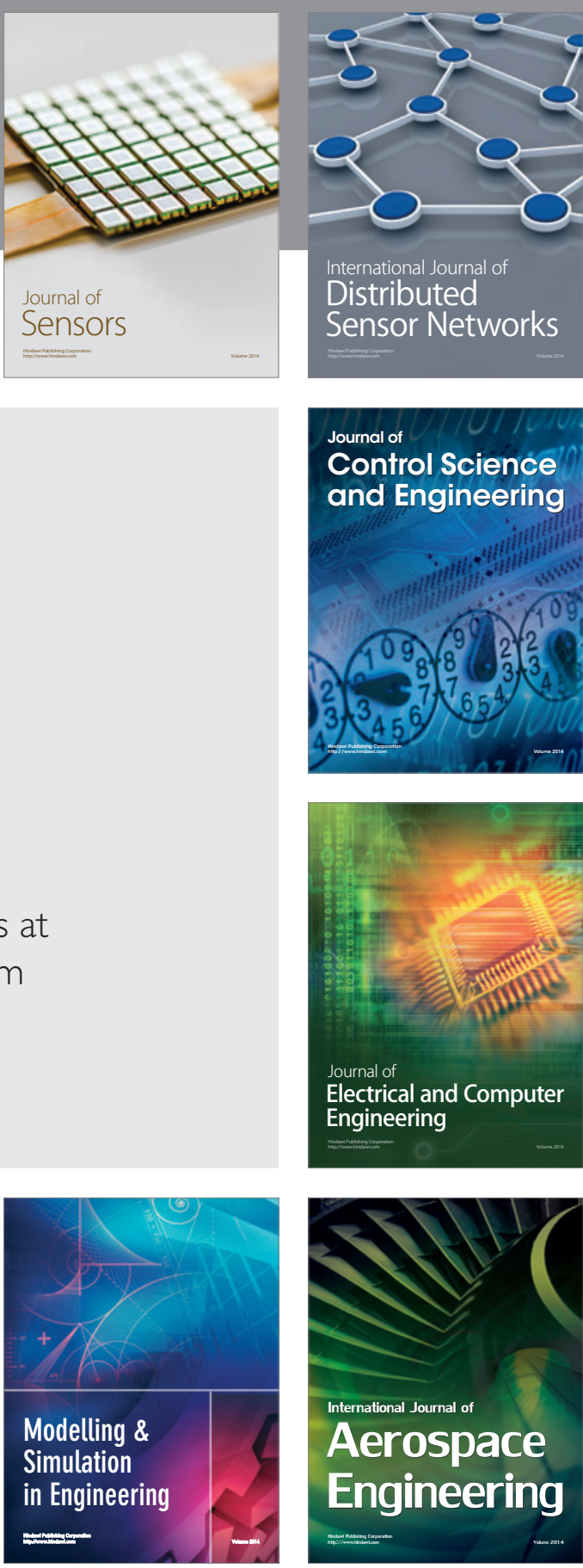

International Journal of

Distributed

Sensor Networks

Journal of

Control Science

and Engineering
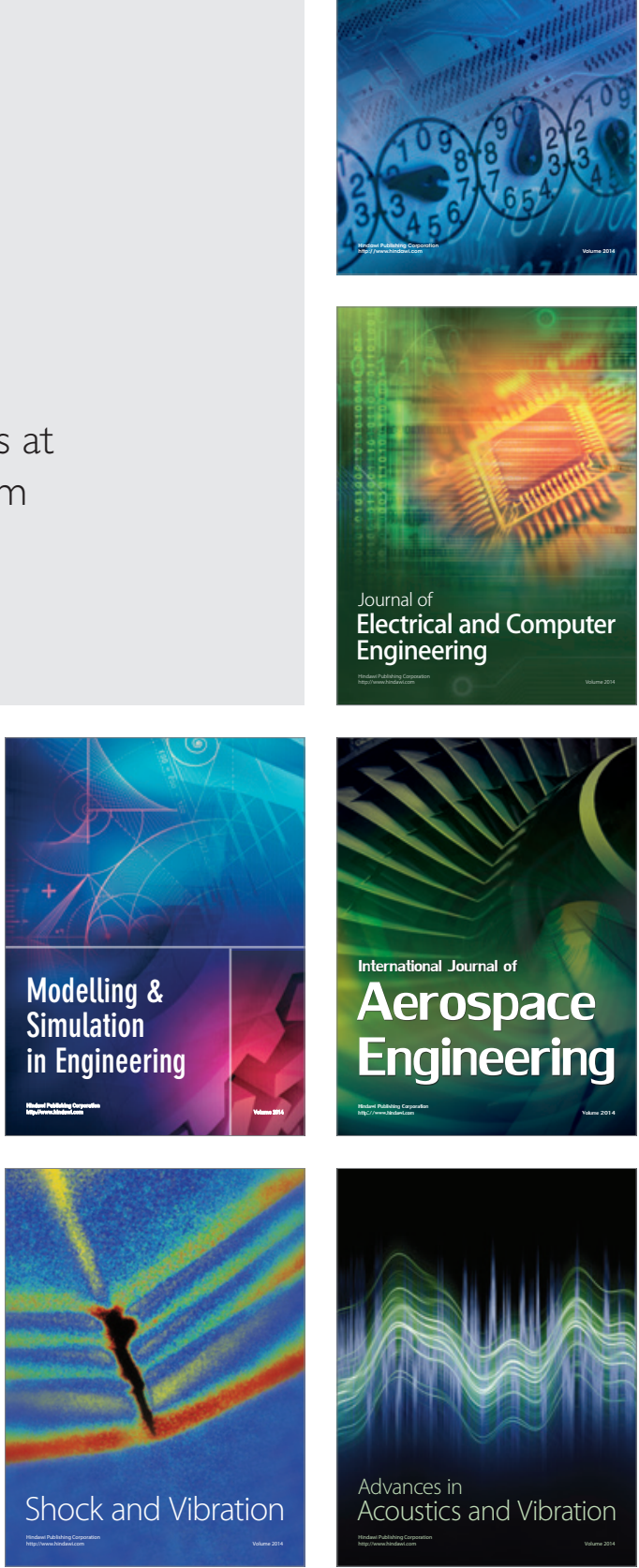